\title{
Optical manipulation of valley pseudospin
}

\author{
Ziliang Ye ${ }^{1,2 \dagger}$, Dezheng Sun ${ }^{1,2 \dagger}$ and Tony F. Heinz ${ }^{1,2 \star}$
}

The coherent manipulation of spin and pseudospin underlies existing and emerging quantum technologies, including quantum communication and quantum computation ${ }^{1,2}$. Valley polarization, associated with the occupancy of degenerate, but quantum mechanically distinct valleys in momentum space, closely resembles spin polarization and has been proposed as a pseudospin carrier for the future quantum electronics ${ }^{3,4}$. Valley exciton polarization has been created in the transition metal dichalcogenide monolayers using excitation by circularly polarized light and has been detected both optically ${ }^{5-7}$ and electrically ${ }^{8}$. In addition, the existence of coherence in the valley pseudospin has been identified experimentally'. The manipulation of such valley coherence has, however, remained out of reach. Here we demonstrate all-optical control of the valley coherence by means of the pseudomagnetic field associated with the optical Stark effect. Using below-bandgap circularly polarized light, we rotate the valley exciton pseudospin in monolayer $\mathrm{WSe}_{2}$ on the femtosecond timescale. Both the direction and speed of the rotation can be manipulated optically by tuning the dynamic phase of excitons in opposite valleys. This study unveils the possibility of generation, manipulation, and detection of the valley pseudospin by coupling to photons.

The broken inversion symmetry in monolayer transition metal dichalcogenide (TMDC) crystals in the semiconducting $\mathrm{MX}_{2}$ $(\mathrm{M}=\mathrm{Mo}, \mathrm{W} ; \mathrm{X}=\mathrm{S}, \mathrm{Se})$ family gives rise to a nontrivial Berry phase at the $K$ and $K^{\prime}$ valleys in momentum space ${ }^{10}$, where the optically accessible direct bandgap occurs in these materials ${ }^{11,12}$. This Berry phase, together with the angular momentum of the atomic orbitals, leads to different optical selection rules for the two valleys: excitons in the $\mathrm{K}$ valley couple to left-circularly polarized photons, while excitons in the $\mathrm{K}^{\prime}$ valley couple to right-circularly polarized photons, thus permitting the optical generation of valley polarization through control of the helicity of light ${ }^{5-7}$. Moreover, when a TMDC monolayer is excited with linearly polarized light, a coherent superposition state is established between the $\mathrm{K}$ and $\mathrm{K}^{\prime}$ valley excitons ${ }^{9}$. Such a coherent state can, in principle, be manipulated by lifting the energy degeneracy between valleys through the breaking of time-reversal symmetry in the material. In this regard, d.c. magnetic fields have been applied to achieve a valley splitting of a few $\mathrm{meV}^{13-16}$. Circularly polarized light also breaks time-reversal symmetry, and researchers have used it to create larger valley splittings, corresponding to pseudomagnetic fields up to $60 \mathrm{~T}$ (refs 17,18). In addition, this optical approach permits us to access the ultrafast timescale: in this work, we demonstrate the coherent manipulation of the valley pseudospin on the femtosecond timescale.

The optical Stark effect has been applied to manipulate the spin/pseudospin in quantum systems, such as atomic quantum gases $^{19}$, quantum dots ${ }^{20,21}$, and III-V quantum wells ${ }^{22}$. Here we utilize this strong pseudomagnetic field in monolayer $\mathrm{WSe}_{2}$ to rotate the valley pseudospin in the superposition plane of $\mathrm{K}$ and $\mathrm{K}^{\prime}$ valley polarizations (Fig. 1). We start by exciting the monolayer with near-resonant linearly polarized light to generate a coherent superposition state. In the Bloch sphere representation, this is equivalent to a pseudospin lying in the equatorial plane, with the $|K\rangle$ and $\left|K^{\prime}\right\rangle$ states pointing towards the north and south poles, respectively. Following this coherent excitation, we apply a belowgap circularly polarized control pulse to lift the valley energy degeneracy by $\hbar \Delta \omega$. The induced change in the transition energy of the two valleys leads to different oscillation frequencies for excitons at $\mathrm{K}$ and $\mathrm{K}^{\prime}$ valleys and, accordingly, to the development of a dynamic phase difference $\Delta \phi \sim \Delta \omega \Delta t$ for a control pulse of duration $\Delta t$. We can describe the effect of this phase evolution as a rotation of the valley pseudospin in the equatorial plane of the Bloch sphere by an angle $\Delta \phi$.

We first use transient reflectivity spectroscopy to characterize the breaking of the valley degeneracy by the optical Stark effect, as previously explored in refs 17 and 18. (See Methods for details concerning our measurements.) As shown in Fig. 1c, the exciton peak in monolayer $\mathrm{WSe}_{2}$ is blue-shifted by about $2 \pm 0.5 \mathrm{meV}$ for a circularly polarized control pulse with a fluence of $1 \mathrm{~mJ} \mathrm{~cm} \mathrm{c}^{-2}$ (at the sample after correcting for the influence of the substrate) with a red-detuning of $160 \mathrm{meV}$ from the A exciton. This optical Stark shift, as previously reported ${ }^{17,18}$, occurs only in one valley and induces an energy splitting between the $K$ and $K^{\prime}$ valleys. (The observable absorption peak shift in a pump-probe experiment is smaller than the intrinsic optical Stark shift of $10 \mathrm{meV}$ that would be achieved for a longer control pulse of the same peak intensity. This situation arises because the duration of control pulse is comparable to the coherence time of the excitonic transition, so that averaging over the time-dependent control pulse leads to a reduced shift. We analyse this effect in detail in Supplementary Section IV.) The energy splitting implies a pseudospin beat frequency of $\Delta \omega / 2 \pi=0.48 \mathrm{THz}$. Based on our control pulse duration of $\Delta t \sim 100 \mathrm{fs}$, we expect an induced pseudospin rotation of roughly $0.1 \pi$, comparable to the experimental results presented below.

We track the valley pseudospin in the Bloch sphere (Fig. 1d) through its signature in the polarization of the photoluminescence? Following application of the linearly polarized excitation pulse and of the circularly polarized control pulse, we collect the photoluminescence (PL) signal and analyse its polarization state (Methods). Since the linearly polarized excitation generates valley coherence between excitons in the TMDC monolayer, the PL exhibits a linear polarized component with its axis aligned with the excitation direction. (In the ideal case, the emission would be fully linearly polarized for linearly polarized excitation; dephasing effects reduce the degree of linear polarization, but do not change its orientation.) If the valley exciton pseudospin is rotated in the Bloch sphere by an angle $\Delta \phi$, the axis of the PL polarization will rotate by half of the corresponding angle $\Delta \phi / 2$ (Supplementary Section I). Thus, by measuring the polarization of the PL after application of

\footnotetext{
${ }^{1}$ Departments of Applied Physics and Photon Science, Stanford University, 348 Via Pueblo Mall, Stanford, California 94305, USA. ${ }^{2}$ SLAC National Accelerator Laboratory, 2575 Sand Hill Road, Menlo Park, California 94025, USA. ${ }^{\dagger}$ These authors contributed equally to this work.

*e-mail: tony.heinz@stanford.edu
} 


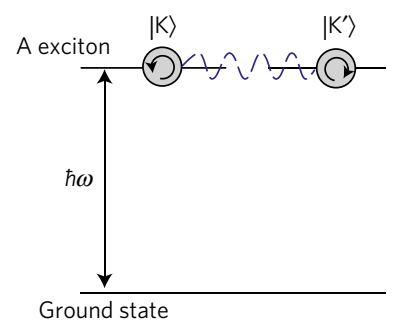

c

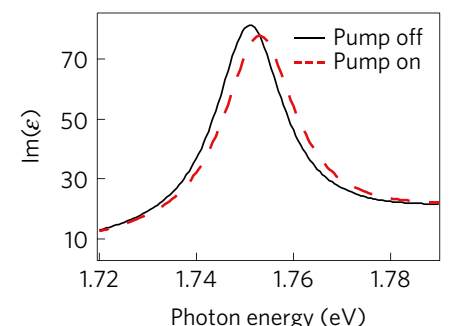

b

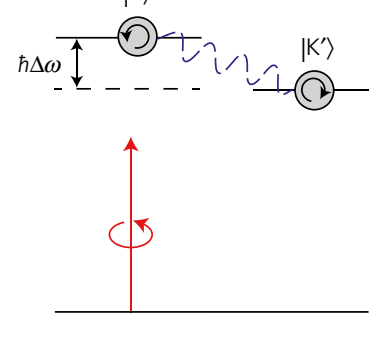

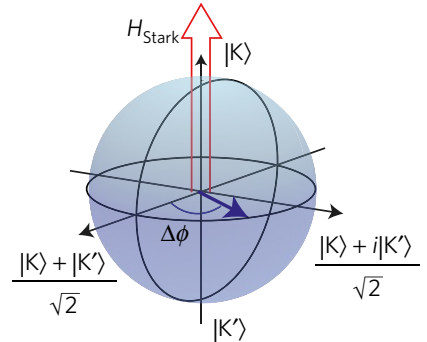

$\sqrt{2}$

$\left|K^{\prime}\right\rangle$

$\underline{|K\rangle+i\left|K^{\prime}\right\rangle}$

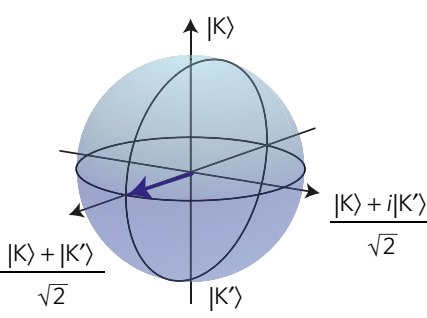

d

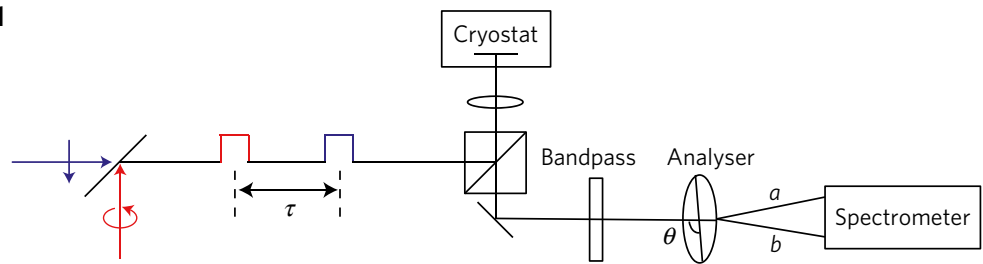

Figure 1 | Schematic representation of all-optical valley pseudospin manipulation. a, A linearly polarized optical pulse excites a coherent superposition of $\mathrm{K}$ and $\mathrm{K}^{\prime}$ excitons at energy $\hbar \omega$. The two equal components in the $\mathrm{K}$ and $\mathrm{K}^{\prime}$ valleys have a fixed phase relationship, as illustrated by the wavy line and by the pseudospin lying in the equatorial plane of the Bloch sphere (right). $\mathbf{b}$. When a strong left-circularly polarized control pulse of below-gap radiation is applied (solid arrow), the exciton transition energy in the K valley is increased by $\hbar \Delta \omega$. While the control pulse is present, a dynamic phase difference $\Delta \phi$ develops between the exciton components in the two valleys. The pseudospin in the Bloch sphere is correspondingly rotated by an angle $\Delta \phi$, just as would occur for an out-of-plane magnetic field (open arrow). c, Results of a transient reflectivity measurement, indicating a valley energy splitting of $2 \pm 0.5$ meV produced by the optical Stark effect with a circularly polarized control pulse. The black solid and red dashed peaks are, respectively, the imaginary part of the fitted dielectric functions with and without the pump pulse. d, Experimental configuration used to generate and monitor the rotation of the valley pseudospin. An ultrafast control pulse is introduced at a controlled time delay $\tau$ after the linearly polarized excitation pulse. The photoluminescence generated by the excitation pulse is spectrally filtered, analysed in terms of its polarization state, and collected by an imaging spectrometer.

our control pulse, we can determine the direction and speed of the pseudospin rotation.

Experimentally, we first examine the influence of the control pulse by detecting the polarized PL using an optical bridge scheme. For linearly polarized excitation along the $x$ direction (Fig. 2), we set the analyser to $\theta=45^{\circ}$ and collect the parallel ( $\boldsymbol{a}$ channel) and perpendicular ( $\boldsymbol{b}$ channel) components of the photoluminescence. The A exciton PL peak in $\mathrm{WSe}_{2}$ is observed at $1.725 \mathrm{eV}$, with a Stokes shift of about $20 \mathrm{meV}$ and a small trion peak at $1.701 \mathrm{eV}$, sitting on a background from localized states and higher-lying transitions. In the absence of the control pulse, the polarization axis of the emission coincides with the excitation direction: the two detection channels then yield identical spectra (Fig. 2, lower). For a leftcircularly polarized control pulse introduced nearly simultaneously with the excitation pulse, the response in the two channels (Fig. 2, upper curves) differs near the A peak, with the $\boldsymbol{a}$ channel increasing significantly for photon energies from 1.712 to $1.738 \mathrm{eV}$. This indicates an anticlockwise rotation of the valley pseudospin induced by the control pulse.

We summarize the influence of the polarization rotation by recording the PL intensities $I_{\mathrm{a}}$ and $I_{\mathrm{b}}$ of the A exciton in the two orthogonal detection channels. (The A exciton strength is obtained by fitting the experimental emission spectra and excluding the background contribution.) The rotation-induced PL anisotropy is described by the normalized Stokes parameter $S_{2}=\left(I_{\mathrm{a}}-I_{\mathrm{b}}\right) /$ $\left(I_{\mathrm{a}}+I_{\mathrm{b}}\right)$ for the analyser set at $\theta=45^{\circ}$. As shown in the inset of Fig. 2, $S_{2}$ increases monotonically with the power of the control beam. This trend is expected provided that the induced rotation of the polarization does not exceed $\pi / 4$ for the highest power in our experiment.

To measure the orientation of the polarization of the PL more precisely, we performed measurements in which we scan the analyser angle $\theta$ (Fig. 3, with complete spectra in Supplementary Section V). For arbitrarily polarized light with a linear polarization component, $S_{2}(\theta)$ varies as $\cos \left[2\left(\theta-\theta_{0}\right)\right]$, where $\theta_{0}$ is the angle of the linearly polarized component of the radiation with respect to the excitation direction. In Fig. 3, we show the results for different polarization states of the control pulses. When the control pulse is linearly polarized, $S_{2}$ follows the expected sinusoidal variation with $\theta_{0}=0$, corresponding to no rotation of the valley pseudospin. However, for a left-circularly polarized control pulse (blue squares), the angular dependence is clearly shifted, corresponding to $\theta_{0}=0.12 \pi=22^{\circ}$ or a $44^{\circ}$ anticlockwise rotation of the valley pseudospin in the Bloch sphere. For a right-circularly polarized control pulse (red diamonds), the angular dependence is shifted in the opposite direction by $\theta_{0}=-0.11 \pi$. This result directly confirms that the direction of polarization rotation of the PL (and, hence, of the underlying valley pseudospin) is determined by the helicity of the circularly polarized control pulse. We note that the amount of pseudospin rotation, while constrained here by our detection scheme, should readily cover the full $2 \pi$ range. (The maximal rotation is limited by two-photon PL induced by the control pulse, which complicates the detection of one-photon PL generated by the excitation pulse. In the future, the two-photon PL could, for example, be avoided by limiting the control photon energy to less than half of the exciton transition energy.)

In addition to the rotation of the PL polarization, we also observe a reduction in the maximum value of $S_{2}$. In contrast, when the control pulse either precedes the excitation pulse or is linearly polarized, we find both the orientation of the polarization and the magnitude of $S_{2}$ remain unchanged. The observed depolarization effect under pseudospin rotation can be understood as the consequence of the incoherent integration of the PL in both the temporal and spatial domains (Supplementary Section II); no additional pump-induced decoherence processes need to be invoked.

By varying the delay time of the control pulse after the excitation pulse, we can also directly probe the dynamics of intervalley coherence (Fig. 4). For each choice of excitation-control delay time, 


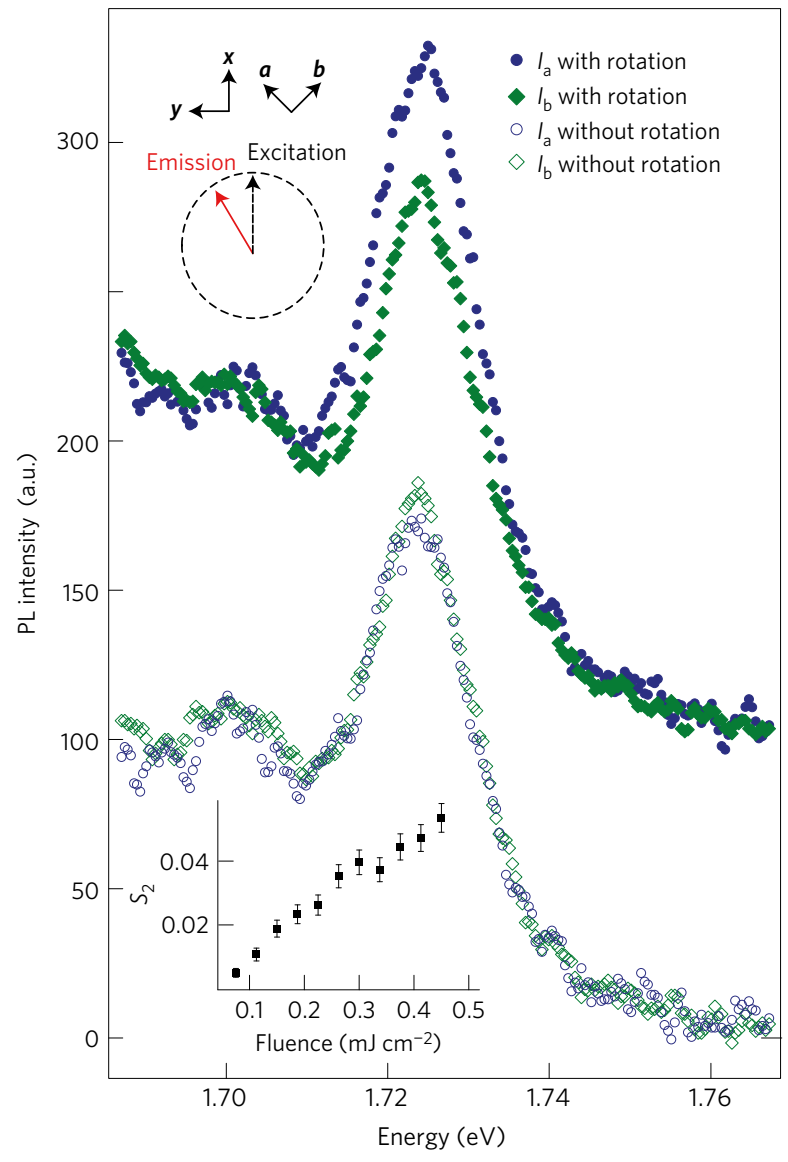

Figure 2 | Photoluminescence spectra for both the rotated (upper) and unrotated (lower) valley pseudospin. The emission both parallel ( $\boldsymbol{a}$ channel, blue dots) and perpendicular ( $\boldsymbol{b}$ channel, green diamonds) to the analyser is collected. Here the analyser is oriented at an angle of $\theta=45^{\circ}$ with respect to the linearly polarized excitation. For a slight time delay of the control pulse with respect to the excitation pulse ( $\tau=50 \mathrm{fs}$ ), the PL of the $\boldsymbol{a}$ channel is clearly stronger than that of the $\boldsymbol{b}$ channel, indicating rotation of the valley pseudospin. For the control pulse preceding the excitation pulse ( $\tau=-200 \mathrm{fs}$ ), no rotation is observed under the same conditions. The PL peaks at $1.701 \mathrm{eV}$ arise from trion states, which do not exhibit intervalley phase coherence and are unaffected by the control pulse. The inset shows the monotonic increase of $S_{2}$ with the fluence of the control pulse. The error bars correspond to the uncertainty from the photon counting statistics of the PL.

we characterize the degree of coherence by the value of $S_{2}\left(\theta=45^{\circ}\right)$. We observe that $S_{2}$ reaches a peak around $50 \mathrm{fs}$, corresponding to the maximum pseudospin rotation in Fig. 3, and decays away within $800 \mathrm{fs}$. This fast response indicates that the intervalley decoherence time $T_{2}$ in monolayer $\mathrm{WSe}_{2}$ lies in the range of hundreds of femtoseconds. If we switch the helicity of the control pulse, the delay dependence of $S_{2}$ is inverted, in agreement with the rotation picture described above.

To analyse the dynamics of valley coherence and control in more detail, we introduce a quantum beat model of the process. For simplicity, we assume instantaneous excitation of the superposition state along the $x$ direction at time $t=0$. We then find that the PL emission along the $\theta$ direction is $I_{\theta}(t) \propto \mathrm{e}^{-t / T_{1}}$ $\left(\rho_{11}^{0}+\rho_{22}^{0}\right)+2 \mathrm{e}^{-t / T_{2}} \rho_{12}^{0} \cos \left(\phi_{1}(t)-\phi_{2}(t)-2 \theta\right)$ (Supplementary Section I). Here $\rho_{11}$ and $\rho_{22}$ are the diagonal elements of the density matrix associated, respectively, with excited states at $\mathrm{K}, \mathrm{K}^{\prime}$ valleys and the ground state, and $\rho_{12}$ is the corresponding offdiagonal element. $T_{1}$ is the exciton population lifetime; $T_{2}$, the

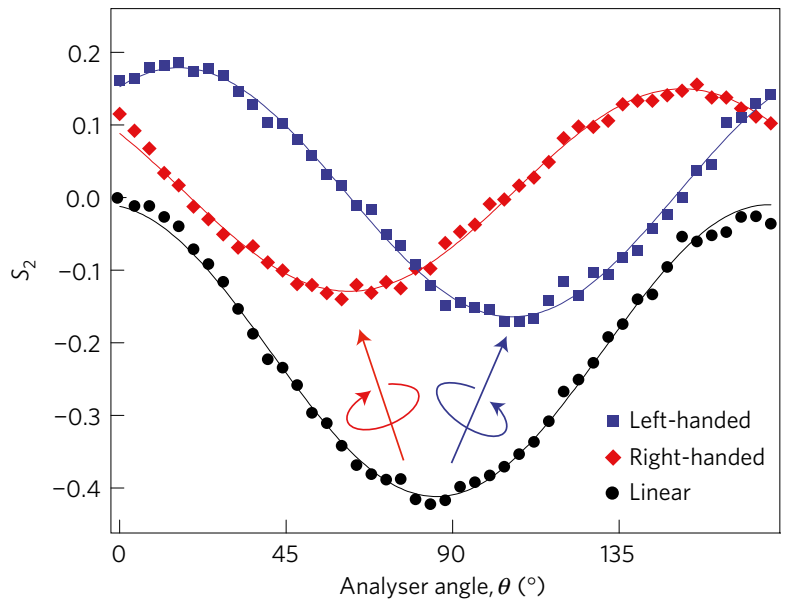

Figure 3 | The rotation of the valley pseudospin determined by the orientation of the polarization of the photoluminescence. As the analyser orientation $\theta$ is scanned over $180^{\circ}$, we collect both $\boldsymbol{a}$ (parallel) and $\boldsymbol{b}$ (perpendicular) channels to determine $S_{2}(\theta)$. When the control pulse is linearly polarized (black dots), $S_{2}$ follows $\cos 2\left(\theta-\theta_{0}\right)$ with $\theta_{0}=0$ (black line), that is, without any discernible angular shift. For a right-circularly polarized control beam, the emission shifts to $\theta_{0}=-0.11 \pi \approx-20^{\circ}$ (red diamonds), corresponding to a clockwise rotation of the pseudospin by $40^{\circ}$. For a left-circularly polarized control beam, the polarization shifts in the opposite direction to $\theta_{0}=0.12 \pi$ (blue squares). The solid curves are sinusoidal fits. For clarity, the $S_{2}$ for the linearly polarized control beam is offset by -0.2 .

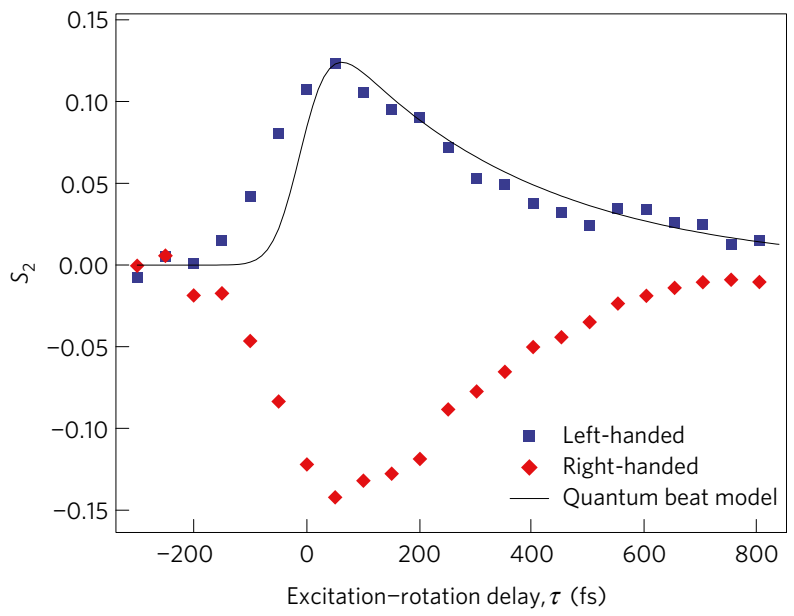

Figure 4 | Determination of the intervalley decoherence time $T_{2}$ from the measurement of the dependence of the rotation-induced PL anisotropy $S_{2}$ on the excitation-control delay time $\boldsymbol{\tau}$. For a left-circularly polarized control pulse, the pseudospin is rotated anticlockwise, leading to an increased value of $S_{2}$ (blue squares). The response peaks for delay of $\tau \sim 50 \mathrm{fs}$ and disappears after $\tau \sim 800 \mathrm{fs}$. The experimental result can be fitted to the predictions of a simple quantum beat model (black line), yielding an intervalley decoherence time $T_{2}=350 \mathrm{fs}$. For the opposite circularly polarized state of the control pulse, the response is inverted (red diamonds), as expected for a clockwise rotation of the valley pseudospin.

intervalley decoherence time; and $\phi_{i}(t)$ (for $i=1,2$ ), the dynamic phase, proportional to the time integral of the time-varying excitedstate energy $\mathrm{E}_{i}(t)$. Since we do not time-resolve the PL in our experiments, we compute the delay dependence of the Stokes parameter $S_{2}$ from the expression for $I_{\theta}(t)$ after an appropriate integration over the whole emission time (Fig. 4, solid line). To apply this model, we use the known duration of the control pulse 
and the fact that the maximum value of $S_{2}$ is 0.20 in the absence of a control pulse. This information allows us to estimate the ratio between $T_{1}$ and $T_{2}$, leaving only two independent parameters in the model (Supplementary Section I). (We note that the partial degree of linear polarization has several possible origins, including intervalley scattering induced by defects/impurities, intravalley scattering, and the exchange interaction between excitons ${ }^{23-25}$. Within our model, we neglect possible initial depolarization effects in creating the valley excitation, in accord with the near-resonant character of our optical excitation.) The two independent parameters are the intervalley decoherence time $T_{2}$, and the optical Stark shift. We obtain a match to experiment for $T_{2}=(350 \pm 50) \mathrm{fs}$, a value comparable to the decoherence time measured by recent photonecho experiments ${ }^{26-28}$. Moreover, the fitted Stark shift also agrees with the value determined by our frequency-domain measurements (Supplementary Sections III and IV).

In conclusion, we have demonstrated the possibility of ultrafast rotation of the exciton valley pseudospin in monolayer $\mathrm{WSe}_{2}$, with control of the rate and rotation angle. Our scheme for valley pseudospin manipulation should also extend to the recently discovered quantum-confined states of excitons in TMDC layers ${ }^{29-33}$. For these localized excitonic states, the valley pseudospin degree of freedom is expected to be well preserved ${ }^{34}$, but their lifetimes are far longer than for the free excitons examined in this work. In addition, full control of valley pseudospin could be achieved by means of stimulated Raman adiabatic passage in a real or pseudomagnetic field. More generally, excitons in TMDC layers play a central role in emerging optoelectronic applications of twodimensional van der Waals materials and their heterostructures. The ability to manipulate valley coherence provides access to a new degree of freedom for future quantum devices.

Note added in proof: After submission of our manuscript, results demonstrating the rotation of the valley pseudospin by static magnetic fields were reported by other researchers ${ }^{35,36}$.

\section{Methods}

Methods, including statements of data availability and any associated accession codes and references, are available in the online version of this paper.

\section{Received 24 January 2016; accepted 17 August 2016; published online 19 September 2016}

\section{References}

1. Kane, B. E. A silicon-based nuclear spin quantum computer. Nature 393, 133-137 (1998)

2. Wolf, S. A. et al. Spintronics: a spin-based electronics vision for the future. Science 294, 1488-1495 (2001).

3. Xu, X., Yao, W., Xiao, D. \& Heinz, T. F. Spin and pseudospins in layered transition metal dichalcogenides. Nat. Phys. 10,343-350 (2014).

4. Gorbachev, R. V. et al. Detecting topological currents in graphene superlattices. Science 346, 448-451 (2014).

5. Mak, K. F., He, K., Shan, J. \& Heinz, T. F. Control of valley polarization in monolayer $\mathrm{MoS}_{2}$ by optical helicity. Nat. Nanotech. 7, 494-498 (2012).

6. Zeng, H., Dai, J., Yao, W., Xiao, D. \& Cui, X. Valley polarization in $\mathrm{MoS}_{2}$ monolayers by optical pumping. Nat. Nanotech. 7, 490-493 (2012).

7. Cao, T. et al. Valley-selective circular dichroism of monolayer molybdenum disulphide. Nat. Commun. 3, 887 (2012).

8. Mak, K. F., McGill, K. L., Park, J. \& McEuen, P. L. The valley Hall effect in $\mathrm{MoS}_{2}$ transistors. Science 344, 1489-1492 (2014).

9. Jones, A. M. et al. Optical generation of excitonic valley coherence in monolayer WSe $\mathrm{W}_{2}$. Nat. Nanotech. 8, 634-638 (2013).

10. Xiao, D., Liu, G.-B., Feng, W., Xu, X. \& Yao, W. Coupled spin and valley physics in monolayers of $\mathrm{MoS}_{2}$ and other group-VI dichalcogenides. Phys. Rev. Lett. 108, 196802 (2012)

11. Mak, K. F., Lee, C., Hone, J., Shan, J. \& Heinz, T. F. Atomically thin $\mathrm{MoS}_{2}$ : a new direct-gap semiconductor. Phys. Rev. Lett. 105, 136805 (2010).

12. Splendiani, A. et al. Emerging photoluminescence in monolayer $\mathrm{MoS}_{2}$. Nano Lett. 10, 1271-1275 (2010).
13. Srivastava, A. et al. Valley Zeeman effect in elementary optical excitations of monolayer $\mathrm{WSe}_{2}$. Nat. Phys. 11, 141-147 (2015).

14. Aivazian, G. et al. Magnetic control of valley pseudospin in monolayer $\mathrm{WSe}_{2}$. Nat. Phys. 11, 148-152 (2015).

15. MacNeill, D. et al. Breaking of valley degeneracy by magnetic field in monolayer $\mathrm{MoSe}_{2}$. Phys. Rev. Lett. 114, 037401 (2015).

16. Li, Y. et al. Valley splitting and polarization by the Zeeman effect in monolayer $\mathrm{MoSe}_{2}$. Phys. Rev. Lett. 113, 266804 (2014).

17. Sie, E. J. et al. Valley-selective optical Stark effect in monolayer $\mathrm{WS}_{2}$. Nat. Mater. 14, 290-294 (2014).

18. Kim, J. et al. Ultrafast generation of pseudo-magnetic field for valley excitons in WSe ${ }_{2}$ monolayers. Science 346, 1205-1208 (2014).

19. Lim, J., Lee, H.-G., Lee, S., Park, C.-Y. \& Ahn, J. Ultrafast Ramsey interferometry to implement cold atomic qubit gates. Sci. Rep. 4, 5867 (2014).

20. Berezovsky, J., Mikkelsen, M. H., Stoltz, N. G., Coldren, L. A. \& Awschalom, D. D. Picosecond coherent optical manipulation of a single electron spin in a quantum dot. Science 320, 349-352 (2008).

21. Press, D., Ladd, T. D., Zhang, B. \& Yamamoto, Y. Complete quantum control of a single quantum dot spin using ultrafast optical pulses. Nature 456, 218-221 (2008).

22. Gupta, J. A., Knobel, R., Samarth, N. \& Awschalom, D. D. Ultrafast manipulation of electron spin coherence. Science 292, 2458-2461 (2001).

23. Yu, H., Liu, G.-B., Gong, P., Xu, X. \& Yao, W. Dirac cones and Dirac saddle points of bright excitons in monolayer transition metal dichalcogenides. Nat. Commun. 5, 3876 (2014).

24. Wu, F., Qu, F. \& MacDonald, A. H. Exciton band structure of monolayer $\mathrm{MoS}_{2}$ Phys. Rev. B 91, 075310 (2015).

25. Qiu, D. Y., Cao, T. \& Louie, S. G. Nonanalyticity, valley quantum phases, and lightlike exciton dispersion in monolayer transition metal dichalcogenides: theory and first-principles calculations. Phys. Rev. Lett. 115, 176801 (2015).

26. Moody, G. et al. Intrinsic homogeneous linewidth and broadening mechanisms of excitons in monolayer transition metal dichalcogenides. Nat. Commun. 6, 8315 (2015).

27. Hao, K. et al. Direct measurement of exciton valley coherence in monolayer $\mathrm{WSe}_{2}$. Nat. Phys. 12, 677-682 (2016).

28. Dey, P. et al. Optical coherence in atomic-monolayer transition-metal dichalcogenides limited by electron-phonon interactions. Phys. Rev. Lett. 116, 127402 (2016).

29. Srivastava, A. et al. Optically active quantum dots in monolayer $\mathrm{WSe}_{2}$. Nat. Nanotech. 10, 491-496 (2015).

30. Koperski, M. et al. Single photon emitters in exfoliated WSe $\mathrm{W}_{2}$ structures. Nat. Nanotech. 10, 503-506 (2015).

31. He, Y.-M. et al. Single quantum emitters in monolayer semiconductors. Nat. Nanotech. 10, 497-502 (2015).

32. Chakraborty, C., Kinnischtzke, L., Goodfellow, K. M., Beams, R. \& Vamivakas, A. N. Voltage-controlled quantum light from an atomically thin semiconductor. Nat. Nanotech. 10, 507-511 (2015).

33. Tonndorf, P. et al. Single-photon emission from localized excitons in an atomically thin semiconductor. Optica 2, 347-352 (2015).

34. Liu, G.-B., Pang, H., Yao, Y. \& Yao, W. Intervalley coupling by quantum dot confinement potentials in monolayer transition metal dichalcogenides. New J. Phys. 16, 105011 (2014).

35. Schmidt, R. et al. Magnetic-field-induced rotation of polarized light emission from monolayer WS $\mathrm{W}_{2}$. Phys. Rev. Lett. 117, 077402 (2016).

36. Wang, G. et al. Control of exciton valley coherence in transition metal dichalcogenide monolayers. Preprint at http://arxiv.org/abs/1606.02051 (2016).

\section{Acknowledgements}

The authors would like to acknowledge technical assistance from X. X. Zhang and S. K. Kim. This work was supported by the Department of Energy, Office of Science, Basic Energy Sciences, Materials Sciences and Engineering Division, under Contract DE-AC02-76SF00515 and by the W. M. Keck Foundation and the Gordon and Betty Moore Foundation's EPiQS Initiative through Grant No. GBMF4545 (T.F.H.)

\section{Author contributions}

Z.Y. and T.F.H. conceived the project. Z.Y. and D.S. performed the experiment. Z.Y., D.S. and T.F.H. analysed the data, developed the model, and wrote the manuscript together.

\section{Additional information}

Supplementary information is available in the online version of the paper. Reprints and permissions information is available online at www.nature.com/reprints. Correspondence and requests for materials should be addressed to T.F.H.

\section{Competing financial interests}

The authors declare no competing financial interests. 


\section{Methods}

Sample preparation and transient reflectivity measurements. We mechanically exfoliated monolayer WSe, samples from bulk crystals onto silicon substrates covered by a $270-\mathrm{nm} \mathrm{SiO}_{2}$ layer. The sample dimensions exceeded $5 \times 5 \mu \mathrm{m}^{2}$, permitting the excitation and control beams to be fully confined to the monolayer. The samples were characterized by both Raman and PL spectroscopy. All experiments were performed at a temperature of $20 \mathrm{~K}$.

We determined the optical Stark shift induced by the control pulse in the frequency domain by means of transient reflectivity measurements ${ }^{17,18}$. The spectra were obtained in a pump-probe configuration using a reflection geometry at zero time delay. The photon energy of the circularly polarized control (pump) pulse here and in the following experiments was chosen to be $1.57 \mathrm{eV}$, approximately $160 \mathrm{meV}$ below the A exciton transition. The induced response was probed by an ultrafast supercontinuum pulse with the same circular polarization, covering a photon energy range from $1.71 \mathrm{eV}$ to $1.79 \mathrm{eV}$. The required pulses were derived from the second harmonic of an amplified mode-locked fibre laser (Impulse, Clarke-MXR) operating at a wavelength of $1.03 \mu \mathrm{m}$. The $1.57 \mathrm{eV}$ pulses were generated by a non-collinear optical parametric amplifier (NOPA), while the continuum pulses were produced by focusing fibre laser pulses of approximately $1 \mu \mathrm{J}$ energy in an undoped YAG crystal. Both pulses had a full-width at half-maximum of $100 \mathrm{fs}$ calibrated by the rising curve of the transient reflection signal from a gold thin film. The repetition rate for these measurements and for the pseudospin rotation experiments was $1 \mathrm{MHz}$. The pump and probe beams were focused at the sample with respective diameters of $2.1 \mu \mathrm{m}$ and $1.7 \mu \mathrm{m}$. The finite size and temporal duration of the pump pulse reduced the optical Stark shift measured by the probe pulse. (The corresponding corrections are analysed in Supplementary Section IV.) The pump beam was chopped at $700 \mathrm{~Hz}$ and the resultant modulation of the probe beam was measured by a lock-in technique. The reflection contrast spectra with and without pump excitation are presented in Supplementary Section III.

Polarization-resolved photoluminescence measurements. Measurements of the valley pseudospin rotation were performed by means of polarization-resolved photoluminescence in the presence of sub-gap control pulses. The control pulses at a photon energy of $1.57 \mathrm{eV}$ were obtained from a NOPA, as described above, with the desired polarization state defined by a Babinet-Soleil compensator. Excitation pulses were obtained from filtered supercontinuum radiation with a photon energy of $1.81 \mathrm{eV}, 85 \mathrm{meV}$ blue-detuned from the A exciton resonance, to maximize the linearly polarized coherent emission ${ }^{37}$. The fluence of the excitation pulses was limited to $1 \mu \mathrm{J} \mathrm{cm}^{-2}$ to avoid saturation, while the fluence of the control pulses did not exceed $1 \mathrm{~mJ} \mathrm{~cm}^{-2}$. The photoluminescence was recorded over a spectral range determined by a 700-nm longpass and a $750-\mathrm{nm}$ shortpass filters. The polarization characteristics of the PL were analysed by a rotatable half-wave plate, followed by a polarizing beam splitter. Measurement of both of the resulting signals (the $\boldsymbol{a}$ and $\boldsymbol{b}$ channels described above) yielded significant reduction in common-mode noise in the system.

Data availability. The data supporting the plots within this paper and other findings of this study are available from the corresponding author upon request.

\section{References}

37. Wang, G. et al. Giant enhancement of the optical second-harmonic emission of $\mathrm{WSe}_{2}$ monolayers by laser excitation at exciton resonances. Phys. Rev. Lett. 114, 097403 (2015) 